\title{
Gluon content of the nucleon probed with real and virtual photons
}

\author{
P. Aurenche \\ Laboratoire de Physique de Particules, Boîte Postale 909, F-74019 Annecy-le-Vieux, France
}

R. Baier

Fakultät für Physik, Universität Bielefeld, D-4800 Bielefeld 1, Germany

M. Fontannaz

Laboratoire de Physique Théorique et Hautes Énergies, Bâtiment 211, Université de Paris XI, F-91405 Orsay CEDEX, France

\section{J. F. Owens}

Physics Department, Florida State University, Tallahassee, Florida 32306

\author{
M. Werlen
}

Université de Lausanne, Institut de Physique Nucleaire, BSP Dorigny, CH-1015 Lausanne, Switzerland

(Received 29 December 1988)

\begin{abstract}
A common fit is performed to lepton-hadron deep-inelastic scattering and large- $p_{T}$ direct-photon cross sections, using complete beyond-leading-logarithm QCD expressions. For direct-photon cross sections, theoretical uncertainties coming from the renormalization and factorization schemes are removed by applying the principle of minimal sensitivity. We find that the direct-photon data, especially from the recent fixed-target experiments, strongly constrain the gluon distribution function, whereas the deep-inelastic data determine the quark distribution functions and constrain the value of $\Lambda_{\overline{\mathrm{MS}}}$ ( $\overline{\mathrm{MS}}$ denotes the modified minimal-subtraction scheme). Our analysis is a successful test of perturbative QCD, which provides a coherent and quantitative description of two very different reactions.
\end{abstract}

\section{INTRODUCTION}

It is well recognized that the hadronic reactions producing large- $p_{T}$ direct photons provide remarkable tests of perturbative QCD (Refs. 1 and 2). The several advantages of direct-photon production as a rich source of information on the hard-scattering dynamics of quarks and gluons have often been pointed out in the literature. ${ }^{3}$ They are due to the pointlike coupling of the photons to the quarks. The trigger photon represents the full jet; therefore, no (nonperturbative) decay function enters into the prediction. Furthermore, starting at the leading order only two subprocesses are relevant: namely, the QCD Compton process $q g \rightarrow q \gamma$ and the annihilation process $q \bar{q} \rightarrow g \gamma$. These two subprocesses may even be disentangled by taking cross-section differences of the type $\sigma(p \bar{p} \rightarrow \gamma X)-\sigma(p p \rightarrow \gamma X)$; the valence-quark and gluon properties in the incident particles can then be studied separately.

The calculation of the complete higher-order QCD corrections at order $O\left(\alpha_{s}^{2}\right)$ for direct-photon (DP) production have been performed. ${ }^{4,5}$ Therefore, the road is open to a quantitative comparison between theory and experiment, with the theoretical inputs, such as $\Lambda_{\mathrm{QCD}}$ and the parton distribution functions, defined beyond the leading order.
Currently there is agreement between existing data and the theoretical predictions using, as an approximate input, the leading-order distribution functions (including the gluon distribution) as parametrized by Duke and Owens $^{6}$ (set 1) and $\Lambda_{\mathrm{QCD}}=200 \mathrm{MeV}$. These successful comparisons have been discussed in several places. ${ }^{2,5,7}$

In this paper, we take a different point of view and we try to extract, from DP data (which cover the large- $x$ region) the gluon distribution function in the proton together with the value of $\Lambda_{\mathrm{QCD}}$. For this purpose we mainly study the reaction $p p \rightarrow \gamma X$, which is indeed quite sensitive to the gluon distribution. The quark distributions we take from elsewhere. The best place is deepinelastic scattering (DIS); we fit the recent muon-proton and muon-deuterium data, especially concentrating on the high-statistics measurements by the Bologna-CERNDubna-Munich-Saclay (BCDMS) Collaboration. ${ }^{8,9}$

More precisely, we perform a common fit to DIS and DP data in the following way. The parameters which determine the shape of the gluon distribution, at some reference scale, being kept fixed, we obtain the valence and sea distributions, as well as the value of $\Lambda_{\mathrm{OCD}}$, from DIS data. These distributions are then used to predict DP data. The quality of the fit depends on the gluon shape; varying this shape we study the constraints on the gluon coming from the DP reactions and their compati- 
bility with DIS data. Finally we get as the "best" gluon, the one which describes both sets of data together. For this aim we perform a quantitative study based on $\chi^{2}$ minimization and we put special emphasis on the estimate of error bars on the parameters we try to determine. Unlike what is usually done in large- $p_{T}$ phenomenology, we are not satisfied with good "eyeball" agreement between theory and experiment on semilogarithmic plots.

In Sec. II we give some technical details on the theoretical predictions and on the data we use. Section III is devoted to fits of muon-proton (and deuterium) DIS data ${ }^{8,9}$ without any constraint coming from DP reactions. A common fit to DIS and fixed-target DP data ${ }^{10-12}$ (only proton targets are considered) is performed in Sec. IV, where the "best" gluon is determined, together with the value of $\Lambda_{\mathrm{QCD}}$. In Sec. $\mathrm{V}$ we comment on the comparison with prompt-photon data from CERN ISR (R108, R806, R110) and CERN SppS (UA1, UA2). Section VI discusses the results and the errors attached to the theoretical predictions.

\section{SOME TECHNICAL DETAILS}

Both DP and DIS data are fitted by expressions calculated beyond the leading order. Let us remind the reader of a few important points and definitions.

The calculation of the DP cross section is described in detail in Ref. 5, where one may find a discussion of the renormalization- and factorization-scheme ambiguities inherent in such higher-order corrections; the principle of minimal sensitivity ${ }^{13}$ is used to suppress these ambiguities and to determine the optimum values of the factorization scale $M$ (which appears in the distribution functions) and of the renormalization scale $\mu$ (which is the argument of the strong coupling constant). The optimized cross sections at fixed values of $\sqrt{s}, p_{T}$, and rapidity are compared with the corresponding data.

In order to fit the DIS data, we use the updated version of the computer code of Ref. 14 which includes the $O\left(\alpha_{s}\right)$ QCD corrections. In DIS reactions, the higherorder corrections are small [in the modified minimalsubtraction $(\overline{\mathrm{MS}})$ scheme and with the choice $\mu=M=Q]$. No optimization procedure is performed in this case.

The data fitted are mainly those of the BCDMS (Refs. 8 and 9) and WA70 (Ref. 10) Collaborations. We choose to extract the quark distributions from the BCDMS data obtained in a very-high-statistics DIS experiment. In order to get rid of nuclear effects, we consider only proton and deuterium data. ${ }^{9}$ We shall also briefly discuss the European Muon Collaboration (EMC) data. ${ }^{15}$ The WA70 experiment ${ }^{10}$ measures $p p \rightarrow \gamma X$ at $\sqrt{s}=23 \mathrm{GeV}$. These data have good statistics and well-controlled systematic errors; they are also in excellent agreement with the NA24 results. ${ }^{11}$ A short discussion of recent $p \bar{p} \rightarrow \gamma X$ data of the UA6 Collaboration ${ }^{12}$ is also given.

The DP reaction $p p \rightarrow \gamma X$ is dominated by the subprocess $q g \rightarrow g \gamma$. It is therefore, a direct probe of the gluon content of the proton. The knowledge of the higherorder corrections allows a beyond-leading-order definition of the gluon distribution function; we use throughout the $\overline{\mathrm{MS}}$ (or universal) factorization conven- tion for the gluon. ${ }^{16}$ The kinematical region covered by the CERN SPS experiments $\left(\sqrt{s} \simeq 23 \mathrm{GeV}, 4 \leq p_{T} \leq 7\right.$ $\mathrm{GeV}$ ) corresponds to a Bjorken $x, 0.35 \leq x \leq 0.60$, where the gluon is not constrained by DIS data. [Note that this traditional estimate of the $x$ range covered by the large$p_{T}$ experiments is rather rough: in fact, at $y=0$ the parton momentum integration range goes down to $p_{T} /\left(\sqrt{s}-p_{T}\right)$.] The medium- $p_{T}$ range of DP fixed-target experiments makes them also quite sensitive (because the scale $\mu$ in the coupling constant is small enough) to the value of $\Lambda_{\mathrm{QCD}}$. The special features of ISR, Fermilab, and $p \bar{p}$ collider experiments are also discussed.

Contrary to the DP production, DIS reactions depend on the gluon in an indirect way (through scaling breaking). In the $\overline{\mathrm{MS}}$ factorization scheme, ${ }^{16}$ we can write, in a symbolic way (one quark species),

$$
\begin{aligned}
\frac{F_{2}(Q)}{x}= & {\left[1+\frac{\alpha_{s}(Q)}{2 \pi} f_{q}^{(2)}\right] \otimes q(Q) } \\
& +\frac{\alpha_{s}(Q)}{2 \pi} f_{g}^{(2)} \otimes g(Q),
\end{aligned}
$$

where $q(Q)$ and $g(Q)$ satisfy the Altarelli-Parisi equations

$$
\begin{aligned}
& \frac{\partial q(Q)}{\partial \ln Q}=\frac{\alpha_{s}(Q)}{\pi}\left[P_{q q} \otimes q(Q)+P_{q g} \otimes g(Q)\right], \\
& \frac{\partial g(Q)}{\partial \ln Q}=\frac{\alpha_{s}(Q)}{\pi}\left[P_{g q} \otimes q(Q)+P_{g g} \otimes g(Q)\right],
\end{aligned}
$$

and $P_{i j}=P_{i j}^{(0)}+\left(\alpha_{s} / 2 \pi\right) P_{i j}^{(1)}$ are beyond-leading-order kernels; the symbol $\otimes$ indicates a convolution. From Eq. (1), we see that $F_{2}(Q)$ is not very sensitive to $g(Q)$, which appears as a higher-order correction. Conversely, the data, unless extremely precise, will not strongly constrain $g(Q)$. The gluon distribution can also be extracted from the $Q$ dependence of $F_{2}(Q)$ through Eqs. (2). The BCDMS Collaboration ${ }^{8}$ has used this method to obtain their results for the shape of the gluon distribution. The quark contribution, on the right-hand side of Eq. (2a), may be positive or negative depending on the value of $x$, whereas the gluon contribution is always positive. In the region of interest, where the gluon is non-negligible $(x<0.3)$, there is a delicate compensation between these two contributions and the fitted gluon is sensitive to additional theoretical assumptions ${ }^{17}$ (charm threshold, factorization scheme,...), as well as to small changes in the data for the slope $\partial F_{2} / \partial \ln Q$. Let us also notice that a change in $g(Q)$ may be compensated by a change in the value of $\Lambda_{\mathrm{QCD}}$ (from which there will be some correlation between the gluon and $\Lambda_{\mathrm{QCD}}$ ).

It is important to keep in mind that $F_{2}(Q)$ and $\partial F_{2} / \partial \ln Q$ do not determine locally $g(x, Q)$; only the integrals of $g(x, Q)$ over $x$, from $x$ to 1, appear in Eqs. (1) and (2). Therefore, the large- $x$ region does contribute to $F_{2}$ and $\partial F_{2} / \partial \ln Q$ even for small values of $x$, and it might happen that the DP constraints are not compatible with the fits to DIS data.

All the higher-order calculations used here are performed in the $\overline{\mathrm{MS}}$ renormalization scheme and so $\Lambda_{\mathrm{QCD}}$ 
denotes $\Lambda_{\overline{\mathrm{MS}}}$ (with four flavors). Furthermore, when performing the fits to the DIS data the quark distributions are defined, for technical and historical reasons, in the universal MS factorization scheme.

Let us end this section with a few words about the factorization convention we use when calculating the DP cross section. As discussed in Ref. 5, we do not optimize with respect to the factorization convention and we fix it arbitrarily. We use in this paper two conventions for the quark distributions, the "physical"18 one which defines

$$
x q\left(x, Q^{2}\right)=F_{2}\left(x, Q^{2}\right),
$$

and the universal $\overline{\mathrm{MS}}$ convention ${ }^{16}$ [cf. Eq. (1)]. The results given below for the DP cross sections were obtained with the physical convention Eq. (3). The relationship between the conventions as well as the dependence of our results on the choice mode is discussed in Appendix A.

\section{FIT TO DIS DATA}

We discuss in this section the results of the fits of preliminary BCDMS data. ${ }^{9}$ Only the proton and $F_{2}^{\text {neutron }} / F_{2}^{\text {proton }}$ data are used. Systematic errors, not yet available, are not taken into account. All the data points have $x \geq 0.07$ and $Q^{2} \geq 8.5 \mathrm{GeV}^{2}$. There are 153 points.

In order to be able to calculate DP cross sections, the knowledge of the different quark distributions is necessary. Therefore, a global fit to the structure function $F_{2}$ for proton and neutron targets is performed. The following parametrization is used to describe the distribution functions at $Q_{0}^{2}=2 \mathrm{GeV}^{2}$ :

$$
\begin{aligned}
& \begin{aligned}
x\left[u^{\mathrm{val}}(x)+d^{\mathrm{val}}(x)\right]= & N\left(\eta_{1}, \eta_{2}, \gamma_{u d}\right) \\
& \times x^{\eta_{1}}(1-x)^{\eta_{2}}\left(1+\gamma_{u d} x\right),
\end{aligned} \\
& x d^{\mathrm{val}}(x)=N\left(\eta_{3}, \eta_{4}\right) x^{\eta_{3}}(1-x)^{\eta_{4}}, \\
& 2 x[\bar{u}(x)+\bar{d}(x)+\bar{s}(x)]=A_{s}(1-x)^{\eta_{s}}, \\
& x g(x)=A_{g}(1-x)^{\eta_{g}} .
\end{aligned}
$$

[In the computer code of Ref. 14, the charm is massless. The charm content of the proton is generated through the Altarelli-Parisi equations, Eqs. (2), with a threshold at $Q_{0}^{2}$. This approximation is valid for $Q^{2}>Q_{0}^{2}$. By comparison with an independent computer code by Tung, ${ }^{19}$ we find perfect agreement for the second-order evolved quark and gluon distributions in the $Q^{2}$ range covered by BCDMS data.]

A SU(3)-symmetric sea is assumed. The coefficient $A_{g}$ is fixed by the momentum sum rule and the nine other parameters are determined by a best fit: besides $\Lambda_{\overline{\mathrm{MS}}}$, there are five (two) independent parameters for the valence(sea-) quark distributions and one $\eta_{g}$ for the gluon. We get a rather good fit: ${ }^{20}$

$$
\begin{aligned}
& \eta_{g}=6.35 \pm 1.76, \quad \Lambda_{\overline{\mathrm{MS}}}=0.209 \pm 0.027 \mathrm{GeV}, \\
& \eta_{s}=9.92 \pm 0.72, \quad \chi^{2}=147.5 / 144 \mathrm{DF},
\end{aligned}
$$

showing that the ansatz, Eqs. (4), is flexible enough to describe also the $x$ dependence of $F_{2}$ correctly. [The fit may even be improved by allowing a more general $x$ dependence in the valence parametrizations, Eqs. (4): for example, replacing $1+\gamma_{u d} x$ by $1+\gamma_{u d} x+\gamma_{u d}^{\prime} x^{2}$, the best fit with now ten parameters results in a $\chi^{2}=138.4 / 140$ degrees of freedom (DF), for BCDMS data with $Q^{2}>10$ $\mathrm{GeV}^{2}$ (i.e., for 150 data points). However the gluon shape is not influenced by this modification.] The gluon distribution $x g\left(x, Q^{2}\right)$ for $Q^{2}=10 \mathrm{GeV}^{2}$, is shown in Fig. 1 , together with the Duke-Owens (DO) gluon ${ }^{6}$ (set 1) also at $Q^{2}=10 \mathrm{GeV}^{2}$. Notice the large error on $\eta_{g}$ which explicitly shows that the gluon is indeed not well constrained by DIS data. [Unless otherwise specified, the quoted errors correspond to a variation of one unit of the $\chi^{2}$ from the minimum value (within the quadratic approximation).]

One may ask how the quality of the above fit depends on the input shape. A possibility to modify slightly these shapes consists in taking a different value for the reference scale $Q_{0}$. The same shape as in Eqs. (4), but with $Q_{0}^{2}=5 \mathrm{GeV}^{2}$ leads to

$$
\begin{aligned}
& \eta_{g}=8.37 \pm 1.65, \Lambda_{\overline{\mathrm{MS}}}=0.200 \pm 0.024 \mathrm{GeV}, \\
& \chi^{2}=150.4 / 144 \mathrm{DF} .
\end{aligned}
$$

These results are in good agreement with the values quoted by BCDMS (Ref. 9) and obtained by fitting data not averaged over various beam energies. The corresponding gluon, at $Q^{2}=10 \mathrm{GeV}^{2}$, shown in Fig. 1 and is almost identical to the one in Eq. (5). We also try a more flexible gluon input shape,

$$
x g\left(x, Q_{0}^{2}=2\right)=A_{g}(1-x)^{\eta_{a}} / x^{\eta_{b}},
$$

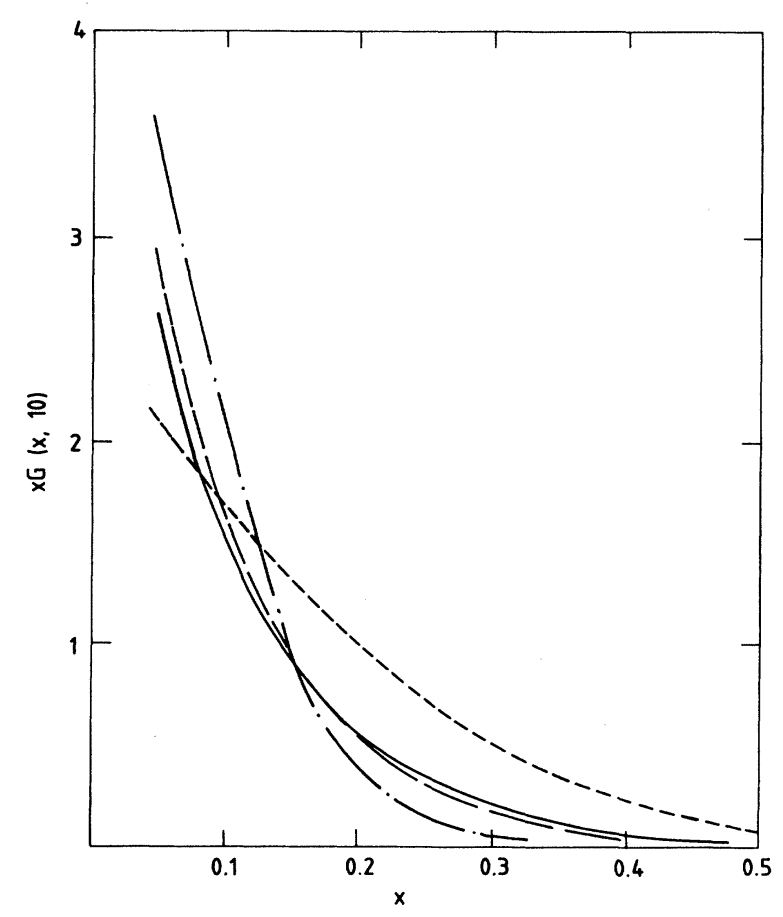

FIG. 1. Gluon distribution as constrained by BCDMS data (see text). Best fit Eq. (5), solid line; best fit Eq. (6), long-dashed line; best fit Eq. (7) dashed-dotted line; Duke-Owens parametrization (set 1), short-dashed line. 
TABLE I. Best fits, with input equations (4), to BCDMS data for different (fixed) values of $\eta_{g}$ at the reference scale $Q_{0}^{2}=2 \mathrm{GeV}^{2}$.

\begin{tabular}{lccccc}
\hline \hline$\eta_{g}$ & $\Lambda_{\overline{\mathrm{MS}}}(\mathrm{MeV})$ & $\chi^{2}$ & $A_{g}$ & $\eta_{s}$ & $A_{s}$ \\
\hline 3.0 & $245.5 \pm 25.1$ & 148.8 & 1.62 & $11.21 \pm 0.54$ & $1.40 \pm 0.07$ \\
3.5 & $236.5 \pm 25.0$ & 148.4 & 1.82 & $11.01 \pm 0.56$ & $1.38 \pm 0.07$ \\
3.8 & $231.9 \pm 24.9$ & 148.2 & 1.95 & $10.88 \pm 0.56$ & $1.37 \pm 0.07$ \\
4.0 & $229.2 \pm 24.9$ & 148.1 & 2.03 & $10.79 \pm 0.57$ & $1.37 \pm 0.07$ \\
4.5 & $223.4 \pm 24.8$ & 147.8 & 2.23 & $10.59 \pm 0.58$ & $1.35 \pm 0.07$ \\
5.0 & $218.6 \pm 24.8$ & 147.6 & 2.43 & $10.39 \pm 0.59$ & $1.34 \pm 0.07$ \\
5.5 & $214.8 \pm 24.7$ & 147.5 & 2.62 & $10.21 \pm 0.59$ & $1.33 \pm 0.06$ \\
6.0 & $211.6 \pm 24.7$ & 147.5 & 2.82 & $10.05 \pm 0.58$ & $1.33 \pm 0.06$ \\
7.0 & $206.7 \pm 24.6$ & 147.7 & 3.21 & $9.79 \pm 0.57$ & $1.33 \pm 0.06$ \\
8.0 & $203.5 \pm 24.5$ & 148.2 & 3.59 & $9.55 \pm 1.52$ & $1.33 \pm 0.13$ \\
9.0 & $200.4 \pm 24.4$ & 149.3 & 3.97 & $9.44 \pm 1.54$ & $1.34 \pm 0.13$ \\
\hline \hline
\end{tabular}

which leads to an improved value of $\chi^{2}\left(\chi^{2}=140.2 / 143\right.$ and $\Lambda_{\overline{\mathrm{MS}}}=0.194 \pm 0.025 \mathrm{MeV}$ ) and to a gluon, at $Q^{2}=10$ $\mathrm{GeV}^{2}$, which is softer than the previous ones (Fig. 1); this improvement of $\chi^{2}$ does not, however, justify the use of the more complicated parametrization (7) and we will not pursue further study of the latter.

Let us consider another example of the poor determination of $\eta_{g}$ from DIS data: the input at $Q_{0}^{2}=2 \mathrm{GeV}^{2}$ is given by Eqs. (4) but, now, we vary $\eta_{g}$ in the interval $3.0 \leq \eta_{g} \leq 9.0$. The results are summarized in Table I and plotted in Figs. 2 and 3. The main observation is that $\chi^{2}$ varies by less than 2 units for this rather large variation with respect to $\eta_{g}$. For $\eta_{g}<5$ some correlation

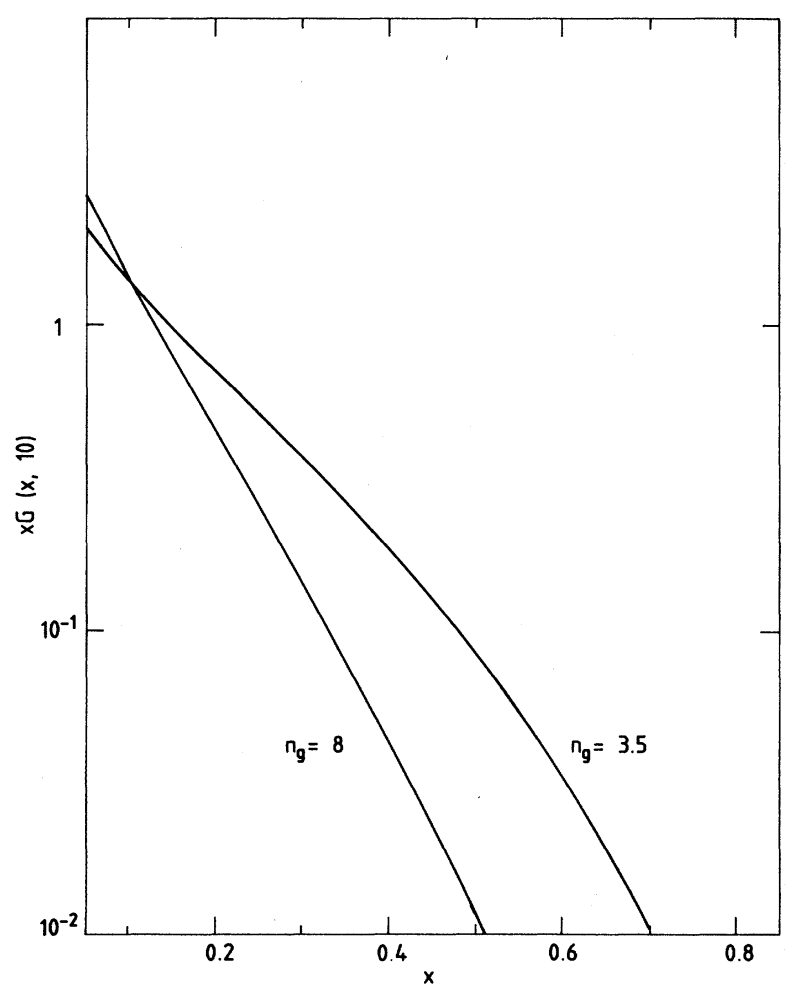

FIG. 2. Examples of gluon distributions corresponding to values of $\chi^{2}$ which differ by less than 1 unit from the best value. with $\Lambda_{\overline{\mathrm{MS}}}$ is to be noticed.

We show in Fig. 2 the comparison between some of the gluon distributions of Table I and we choose two extreme cases which differ by less than one unit of $\chi^{2}$ from the minimum value: as examples we take $\eta_{g}=3.5$ and 8.0. Note that the main differences occur at large values of $x$ as expected. Considering all the fits described previously it appears that the various gluon distributions considered cross one another for $x$ values between 0.1 and 0.2 and that the softer gluons at large $x$ have the largest values at

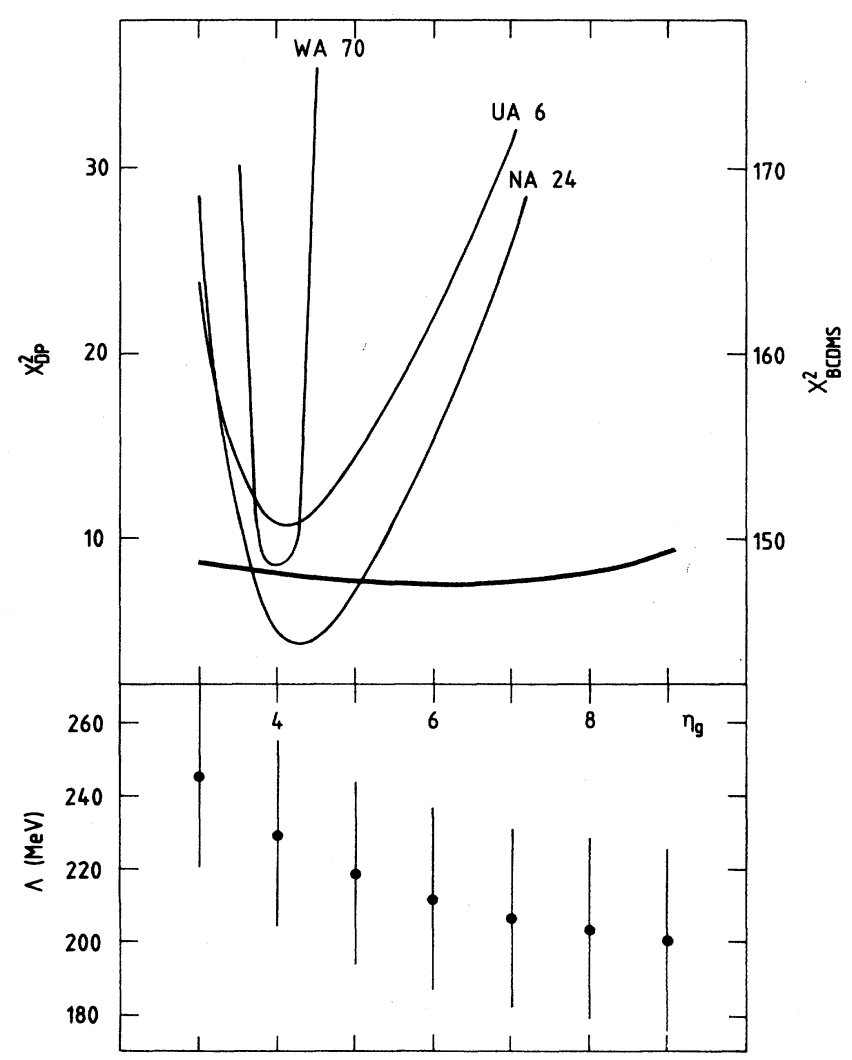

FIG. 3. The $\chi^{2}$ of fits to DIS data (BCDMS) and directphoton cross sections (WA70, UA6, and NA24) as a function of $\eta_{g}$. Variation of the fitted $\Lambda_{\overline{\mathrm{MS}}}$ value. 
small $x$ in agreement with the constraint of the momentum sum rule. As a consequence, the shape of the gluon at large $x$, which is mainly determined by fixed-target DP data will bear on the shape in the $x$ range probed by the DIS data.

The fact that the values of $\chi^{2}$ are almost independent of $\eta_{g}$ is related to the parametrization given in Eqs. (4). We find that the large variations of $\eta_{g}$ are compensated by small changes of the parameters for the quark (mainly sea-quark) distributions and of $\Lambda_{\overline{\mathrm{MS}}}$. The $\chi^{2}$ behavior also depends on the functional form of the input. This may be seen as follows. Instead of starting the evolution in $Q^{2}$ at $Q_{0}^{2}=2 \mathrm{GeV}^{2}$, we may start at $Q_{0}^{2}=5 \mathrm{GeV}^{2}$, for instance. At this value of $Q^{2}$, the original input has already developed a more singular behavior, in what concerns the sea-quark and gluon distributions [e.g., $x g\left(x, Q^{2}=5\right) \propto x^{-0.18}$ at small $\left.x\right]$, whereas the input at $Q_{0}^{2}=5 \mathrm{GeV}^{2}$ does not possess, by definition, these singularities. The latter input leads to a worse $\chi^{2}$ [Eq. (6)] and to a stronger dependence on $\eta_{g}$ (especially at small values of $\eta_{g}$ ) as illustrated in Fig. 4 (solid curve). The variation of $\chi^{2}$ with $\eta_{g}$ also strongly depends on extra constraints on the sea quarks. Keeping, for instance, $\eta_{s}$ fixed at the value $\eta_{s}=9.0$, the $\chi^{2}$ varies by more than 12 units when $\eta_{g}$ is changed between 4 and its best value around 8 (dashed curve in Fig. 4).

It should be stressed that the determination of the valence-quark distributions is remarkably stable under changes of the gluon input: for example, using the parametrizations given in Eqs. (4), the up- and down-quark distributions evaluated at $Q^{2}=5 \mathrm{GeV}^{2}$ are unchanged (less than $0.5 \%$ variation) for $x<0.3$ and increase by

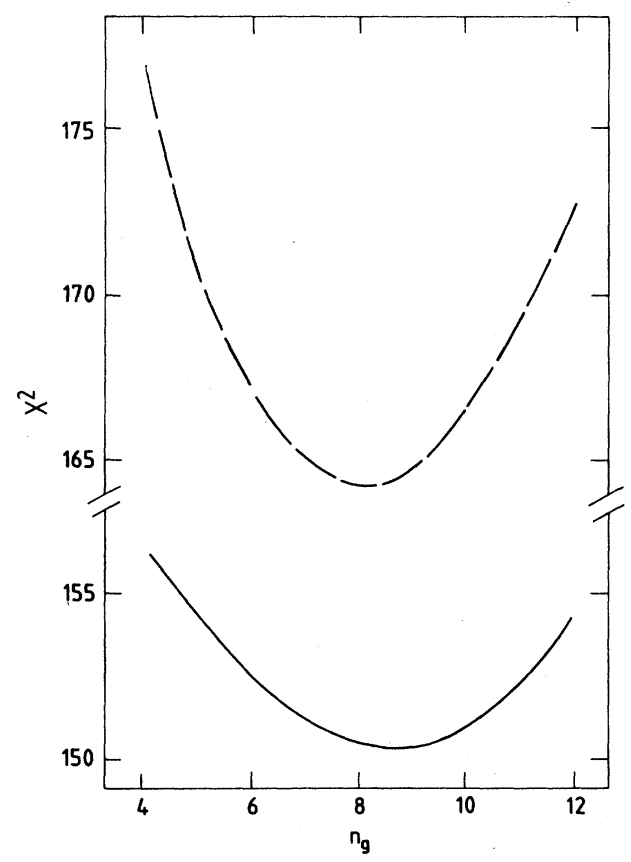

FIG. 4. The $\chi^{2}$, as a function of $\eta_{g}$, of fits to BCDMS data. Dashed line input (4) at $Q_{0}^{2}=5 \mathrm{GeV}^{2}$ and $\eta_{s}=9$. Solid line input (4) at $Q_{0}^{2}=5 \mathrm{GeV}^{2}$. about $2 \%$ at $x=0.8$ when $\eta_{g}$ is varied from 3.0 to 9.0 . We also find that the sea distribution is rather well constrained by the BCDMS data (Table I). This determination is valid for $x \lesssim 0.2$ and we do not try to constrain this distribution at large values of $x$ by a quantitative fit to Drell-Yan data. We just check, using the leadingorder QCD formula for the Drell-Yan cross section, that the Fermilab ${ }^{21}$ and ISR (Ref. 22) data, leaving a free $K$ factor for each experiment, are quite compatible with a value $\eta_{s}=10$.

We end this analysis of DIS data by giving the results of our fit to the EMC data ${ }^{15}$ with the simple input given in Eqs. (4). (With the cutoff $x \geq 0.05, Q^{2} \geq 7 \mathrm{GeV}^{2}$, and $W^{2} \geq 10 \mathrm{GeV}^{2}$ there are 145 data points. In this fit, the relative normalization of the proton and deuterium data is left free.) At $Q_{0}^{2}=2 \mathrm{GeV}^{2}$ we get the parameters

$$
\eta_{g}=9.07 \pm 2.60, \Lambda_{\overline{\mathrm{MS}}}=0.0968 \pm 0.043 \mathrm{GeV}
$$
with

$$
\chi^{2}=191.8 / 135 \mathrm{DF},
$$

again including only the statistical errors. The value of $\eta_{g}$ is compatible, within the errors, with the BCDMS one, although a common fit to these two data samples does not make sense since the corresponding proton structure functions differ, especially in the low- $x$ region. Note also the somewhat lower value of $\Lambda_{\overline{\mathrm{MS}}}$ in agreement with results of the EMC Collaboration. Here also, the error on $\eta_{g}$ is large and the gluon distribution is not well constrained. One should also notice that the value for $\chi^{2} / \mathrm{DF}$ is much larger than those obtained in the BCDMS fits.

An analysis of EMC data similar to the one of BCDMS data reported above can be performed, with similar conclusions; for instance, the dependence of $\chi_{\mathrm{EMC}}^{2}$ on $\eta_{g}$ is also very weak. The high statistics of the BCDMS experiment put, however, more severe constraints on the values of the fitted parameters and make the comparison with DP data more challenging. We shall, therefore, consider only BCDMS data in the following section.

Our conclusion of this short analysis of DIS data is that the shape of the gluon distribution function is not well constrained. This statement, however, partially depends on the parametrization chosen to fit the data; this point is illustrated in Fig. 4 where a strong dependence of $\chi^{2}$ on $\eta_{g}$ is obtained when the sea-quark distribution function is kept fixed. There is, however, no reason to freeze $\eta_{s}$. The Drell-Yan data are indeed not precise enough to constrain $\eta_{s}$ better than the DIS data. We also see that we have to start with an adequate functional form, because otherwise $\chi^{2}$ turns out to be worse and its dependence on $\eta_{g}$ stronger.

For all these reasons, it appears that the parametrization given in Eqs. (4), with all parameters kept free and with $Q_{0}^{2}=2 \mathrm{GeV}^{2}$, is suitable for a good description of DIS data. Therefore, we stick to the latter and to the results of Table I when analyzing DP data.

\section{COMMON FIT TO DIS AND DP DATA}

The WA70 data ${ }^{10}$ that we now study in detail are given in Table II with statistical and systematic errors. 
TABLE II. $E d \sigma(p p \rightarrow \gamma X) / d^{3} p$ as a function of $p_{T}$ $\left(-35<x_{F}<0.45\right)$ from Ref. 10.

\begin{tabular}{lcccc}
\hline \hline $\begin{array}{c}p_{T} \text { range } \\
(\mathrm{GeV})\end{array}$ & $\begin{array}{c}\left\langle p_{T}\right\rangle \\
(\mathrm{GeV})\end{array}$ & $\begin{array}{c}E d \sigma / d^{3} p \\
\left(\mathrm{pb} / \mathrm{GeV}^{2}\right)\end{array}$ & $\begin{array}{c}\text { Stat. } \\
\text { error }\end{array}$ & $\begin{array}{r}\text { Syst. } \\
\text { error }\end{array}$ \\
\hline $4.25-4.50$ & 4.36 & 15.781 & 0.993 & 3.164 \\
$4.50-4.75$ & 4.61 & 8.744 & 0.710 & 1.669 \\
$4.75-5.00$ & 4.86 & 5.720 & 0.539 & 1.083 \\
$5.00-5.25$ & 5.11 & 2.683 & 0.362 & 0.568 \\
$5.25-5.50$ & 5.36 & 1.861 & 0.288 & 0.399 \\
$5.50-6.00$ & 5.70 & 0.463 & 0.104 & 0.123 \\
$6.00-7.00$ & 6.34 & 0.125 & 0.033 & 0.052 \\
\hline \hline
\end{tabular}

In the following, we shall use several methods to extract the QCD parameters $\eta_{g}$ and $\Lambda$ from DP data and check their compatibility with DIS results.

(a) In order to compute the DP cross sections we first proceed in the following way. We take the fits to the BCDMS data given in Table I, for the different values of $\eta_{g}$ and calculate beyond leading order the DP cross sections. The comparison with data is described by the $\chi^{2}$ values (taking only statistical errors into account) which are summarized in Table III for fixed-target DP experiments. Concerning the WA70 data, we observe that the $\chi^{2}$ dependence on $\eta_{g}$ is very peaked and uniquely determines a value of $\eta_{g}$ around 4; this is in contrast with the very flat $\chi_{\text {DIS }}^{2}$ shape. This behavior is very clear in Fig. 3.

The data of the NA24 experiment ${ }^{11}$ are in nice agreement with WA70; we get $\eta_{g}=4.3 \pm 0.4$. The $\chi^{2}$ is, however, less sharply peaked than in the WA70 case, the statistical errors being larger here.

Results from the UA6 Collaboration on $p p \rightarrow \gamma X$ and $p \bar{p} \rightarrow \gamma X$ will provide further interesting tests of QCD and quite valuable information on the gluon content of the proton. For the time being, only $p \bar{p} \rightarrow \gamma X$ results are available, which are less sensitive to the gluon distribution function than those of the $p p \rightarrow \gamma X$ reaction. The results of a preliminary study of these data are given in Table III. The $\chi^{2}$ as a function of $\eta_{g}$ determines a value $\eta_{g}=4.1 \pm 0.4$.

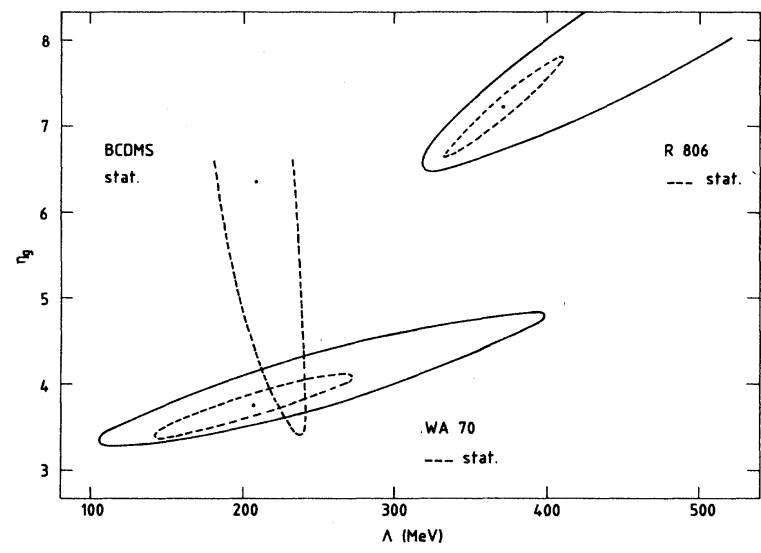

FIG. 5. Error "ellipses" of $\eta_{g}$ (at $Q_{0}^{2}=2 \mathrm{GeV}^{2}$ ) vs $\Lambda$ for direct-photon cross sections (WA70, R806) and deep-inelastic structure functions (BCDMS). The dashed "ellipses" are one standard deviation. The solid "ellipses" take into account systematic errors added in quadrature to statistical errors.

The values of $\eta_{g}^{\mathrm{WA} 70}, \eta_{g}^{\mathrm{NA} 24}$, and $\eta_{g}^{\mathrm{UA6}}$ are compatible within statistical errors (see Fig. 3), which indicates a remarkable coherence between the three fixed-target experiments performed at the CERN SPS. Let us indeed recall that a systematic uncertainty of $20-30 \%$ is given for these experiments.

(b) Let us now decouple the value of $\Lambda_{\overline{\mathrm{MS}}}$ used in the distribution functions and determined by the DIS data from the one used in the strong coupling constant of the DP subprocesses [called $\Lambda\left(\alpha_{s}\right)$ below]. Performing a best fit to WA70 data, we get

$$
\begin{aligned}
& \eta_{g}=3.76 \pm 0.37, \Lambda\left(\alpha_{s}\right)=207.0 \pm 64.0 \mathrm{MeV}, \\
& X_{\text {WA70 }}^{2}=7.8 .
\end{aligned}
$$

The corresponding error "ellipse" $\left(\Delta \chi^{2}=1\right)$ is shown in Fig. 5 together with that obtained from the fit to BCDMS data alone [Eq. (5)]. (The error "ellipses" are obtained without assuming the quadratic approximation.) First we note that the WA70 data are able to give a determination

TABLE III. Best fits to BCDMS (Ref. 9), for fixed values of $\eta_{g}$ at $Q_{0}^{2}=2 \mathrm{GeV}^{2}$, and the correspond-

\begin{tabular}{|c|c|c|c|c|c|}
\hline$\underline{\eta_{g}}$ & $\Lambda_{\overline{\mathrm{MS}}}\left(\begin{array}{c}\mathrm{BCI} \\
(\mathrm{MeV})\end{array}\right.$ & $\chi^{2}$ & $\begin{array}{c}\chi_{\text {WA70 }}^{2} \\
(7 \mathrm{pts}) \\
\left\langle p_{T}\right\rangle \geq 4.36\end{array}$ & $\begin{array}{c}\chi_{\text {NA24 }}^{2} \\
(4 \mathrm{pts}) \\
\left\langle p_{T}\right\rangle \geq 3.75\end{array}$ & $\begin{array}{c}\chi_{\mathrm{UA6}}^{2} \\
(10 \mathrm{pts}) \\
\left\langle p_{T}\right\rangle \geq 4.00 \\
\end{array}$ \\
\hline 3.0 & $245.5 \pm 148.8$ & 146.8 & 146.0 & 27.4 & 23.8 \\
\hline 3.5 & $236.5 \pm 25.0$ & 148.4 & 30.0 & 11.1 & 13.9 \\
\hline 3.8 & $231.9 \pm 24.9$ & 148.2 & 9.3 & 6.5 & 11.5 \\
\hline 4.0 & $229.2 \pm 24.9$ & 148.1 & 8.5 & 4.9 & 10.8 \\
\hline 4.5 & $223.4 \pm 24.8$ & 147.8 & 35.5 & 4.4 & 11.6 \\
\hline 5.0 & $218.6 \pm 24.8$ & 147.6 & 85.0 & 7.0 & 14.5 \\
\hline 6.0 & $211.6 \pm 24.7$ & 147.5 & 202.0 & 16.3 & 22.8 \\
\hline 7.0 & $206.7 \pm 24.6$ & 147.7 & 307.0 & 26.6 & 32.0 \\
\hline 8.0 & $203.5 \pm 24.5$ & 148.2 & 388.0 & 35.6 & 40.0 \\
\hline 9.0 & $200.4 \pm 24.4$ & 129.3 & 448.0 & 42.9 & 48.0 \\
\hline
\end{tabular}
ing $\chi^{2}$ for WA70 (Ref. 10), NA24 (Ref. 11) UA6 (Ref. 12) DP data. $p_{T}$ in GeV. 
of $\Lambda$ consistent with that of BCDMS, although with a larger error. Furthermore, it is remarkable (and encouraging) that the error ellipses do intersect. This is a strong quantitative indication of the validity of QCD beyond leading order. It is interesting to notice that the $\eta_{g}-\Lambda_{\overline{\mathrm{MS}}}$ correlation is quite different in the DIS and DP experiments (Fig. 5). For the determination of these parameters, these two types of reactions are complementary.

As an educated guess of the effect of systematic uncertainties of the WA70 experiment, a fit is performed using the quadratic sum of the statistical and systematic errors of Table II. The resulting "ellipse" is shown in Fig. 5 (solid line).

(c) Let us end this study of the WA70 data by a common fit ( nine-parameter fit [Eq. (4) with the same value of $\Lambda$ in $\alpha_{s}$ and in the distribution functions]) to the latter and to BCDMS data; the agreement between theory and data is described by $\chi_{\text {tot }}^{2}=\chi_{\text {BCDMS }}^{2}+\chi_{\text {WA70. As expected }}^{2}$ from the preceding investigations, the two sets of data are in good agreement with the QCD predictions; the best fit gives the values (the full set of parameters is given in Appendix B)

$$
\begin{aligned}
& \eta_{g}=3.90 \pm 0.11, \quad \Lambda_{\overline{\mathrm{MS}}}=230.0 \pm 17 \mathrm{MeV}, \\
& \chi_{\mathrm{WA} 70}^{2}=7.90, \quad \chi_{\text {tot }}^{2}=156.1 / 151 \mathrm{DF} .
\end{aligned}
$$

The quoted statistical errors correspond to $\Delta \chi_{\text {tot }}^{2}=1$ and the corresponding error "ellipse" is shown in Fig. 6, where we also draw the contour of $90 \%$ C.L. $\left(\Delta \chi_{\text {tot }}^{2}=4.61\right)$. Compared to result (9), we find, as anticipated, a consistent determination of the parameters with much reduced errors.

We now discuss the effects of the WA70 (Ref. 10) systematic uncertainties on the determination of the param-

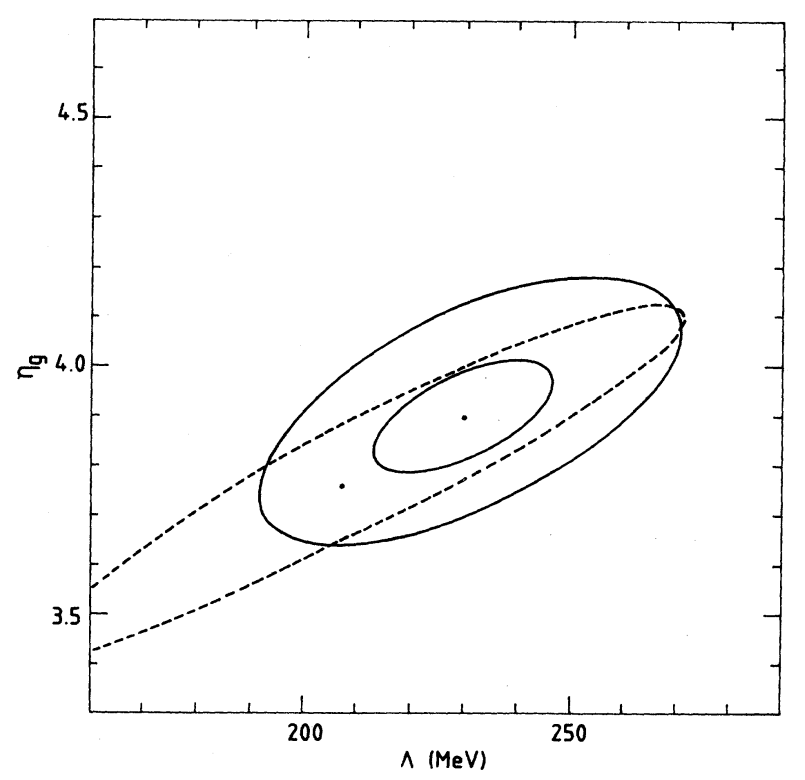

FIG. 6. Errors "ellipses" corresponding to $\Delta \chi_{\text {tot }}^{2}=1$ and $\Delta \chi_{\text {tot }}^{2}=4.61$ (90\% C.L.). The dashed contour is the $\Delta \chi_{\mathrm{WA} 70}^{2}=1$ contour already shown in Fig. 5. eters [Eq. (10)] especially on the value of $\eta_{g}$. In Ref. 10 all systematic errors are added in quadrature and this amounts to $\sim \pm 20 \%$. Since we cannot exclude that some of these errors add linearly, we consider here an overall normalization uncertainty of $\pm 30 \%$. Raising or lowering the normalization of the data by this percentage shifts the central value of $\eta_{g}$ [Eq. (10)] by -0.6 and +0.8 , respectively (the corresponding shift on $\Lambda_{\overline{\mathrm{MS}}}$ amounts to \pm 20 $\mathrm{MeV}$, and $\chi_{\text {WA70 }}^{2}$ is increased by 1.3 and 2.1 , respectively). The errors thus obtained are compatible with those which can be read in Fig. 5 .

We notice that the value of $\Lambda_{\overline{\mathrm{MS}}}$ is essentially determined by the DIS data (this can also be seen from the shape of the ellipse in Fig. 5). Therefore, the systematic errors on $\Lambda_{\overline{\mathrm{MS}}}$ are mainly due to the corresponding ones of the BCDMS data, which lead, following Ref. 9 to errors of $\pm 50 \mathrm{MeV}$. We consider these numbers as our estimate of the systematic uncertainties on the value of $\Lambda_{\overline{\mathrm{MS}}}$ [Eq. (10)]. Within these limits we perform once more the fit to WA70 data: the minimum $\chi^{2}$ values are found at $\eta_{g}=3.7$ for $\Lambda_{\overline{\mathrm{MS}}}=180 \mathrm{MeV}\left(\chi_{\mathrm{WA} 70}^{2}=8.8\right)$ and at $\eta_{g}=4.1$ for $\Lambda_{\overline{\mathrm{MS}}}=280 \mathrm{MeV}\left(\chi_{\text {WA70 }}^{2}=9.2\right)$, i.e., $\Delta \eta_{g}= \pm 0.2$. These errors are small compared to those given above which are induced by WA70 systematics. When these gluon densities are evolved to $Q^{2}=10 \mathrm{GeV}^{2}$ the resulting uncertainties are slightly larger than the statistical ones.

The best gluon from Eq. (10) at $Q^{2}=10 \mathrm{GeV}^{2}$ is displayed in Fig. $7(x<0.4)$ and Fig. $8(x>0.25)$, where the statistical and systematic error bands are indicated. In both figures it is compared to the gluon obtained by Diemoz, Ferroni, Longo, and Martinelli ${ }^{23}$ (DFLM) from fits to CERN-Hamburg-Amsterdam-Rome-Moscow (CHARM) data. ${ }^{24}$ There is consistency over the whole $x$ range between both gluons: for $x>0.25$ the gluon deter-

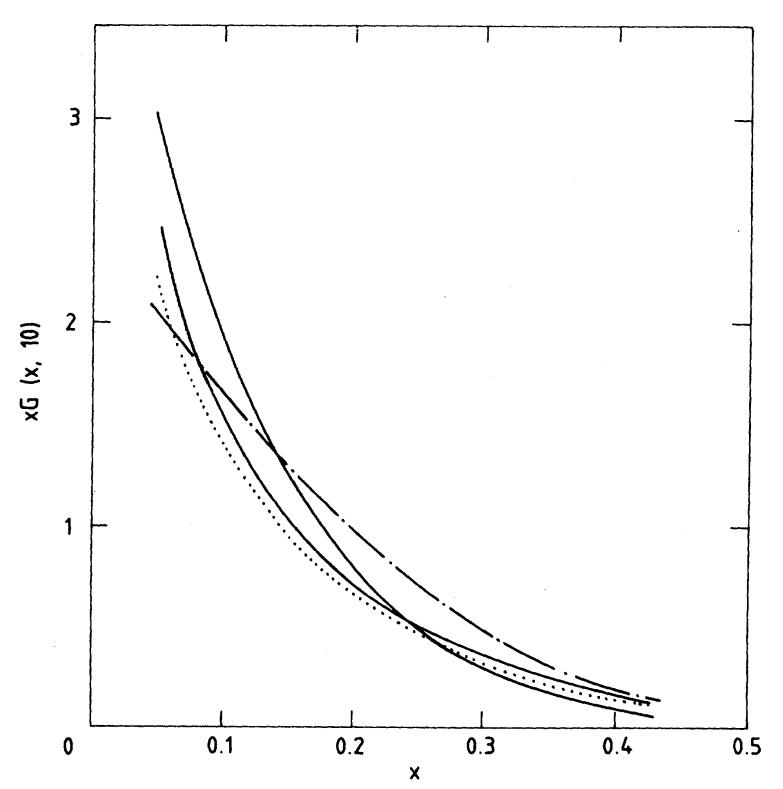

FIG. 7. The gluon distribution from Eq. (10) at $Q^{2}=10 \mathrm{GeV}^{2}$ (dotted curve). The gluon distributions as given by DFLM (Ref. 33) (solid lines) and by DO set 1 (dashed-dotted line). 
mined by DFLM is within the error band discussed before, whereas at small $x$ it is slightly above the "best" gluon of Eq. (10). The gluon distribution derived here is also in good agreement with that used by Martin et al..$^{34}$ in their fits to BCDMS and neutrino data. For reference we also show the leading-order DO set-1 gluon distribution. The WA70 $p p \rightarrow \gamma X$ spectrum compared with the fit based on Eq. (10) is shown in Fig. 9.

\section{COMPARISON WITH OTHER DP DATA}

It is instructive to show the ratio data/theory for various recent $p p$ (Fig. 10) and $p \bar{p}$ (Fig. 11) experiments. We remark that more than 6 orders in magnitude for the cross sections are covered by these plots. The theoretical predictions are obtained with the best fit values (Appendix B). Concerning the $p p$ data, we emphasize good agreement with fixed-target experiments and we note some disagreement with ISR data. ${ }^{25-27}$ This result is similar to the one we obtained in Ref. 5, using the DO set-1 parametrization. However, as shown in Table IV the $\chi^{2}$ values are improved when the beyond-leadingorder evolved structure functions are used to describe all the available DP data. (For the UA1 and UA2 predictions, an isolation cut of $\frac{\delta}{2}=57^{\circ}$ and $\frac{\delta}{2}=45^{\circ}$, respectively, is applied..$^{5}$ In all cases the rapidity smearing of the data is taken into account.)

Concerning the most precise set of ISR data, namely, $\mathrm{R} 806$, the $\chi^{2}$ value obtained in Table IV is astonishingly large. In an attempt to understand if these data can be

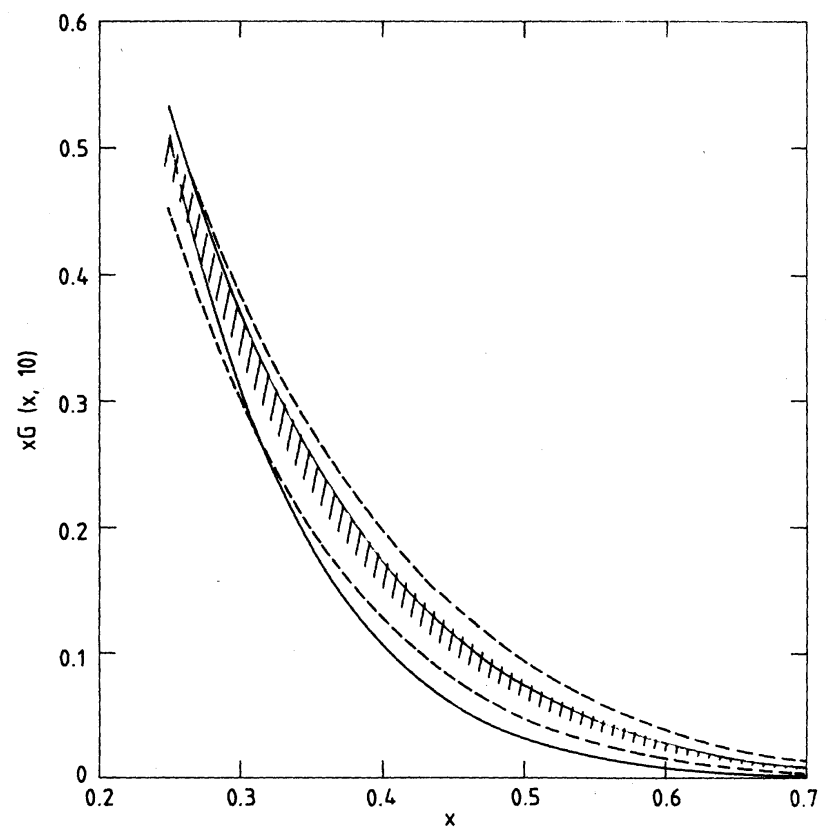

FIG. 8. The gluon distribution from Eq. (10) at $Q^{2}=10$ $\mathrm{GeV}^{2}$. The hatched area (dashed lines) represents the uncertainty due to statistical (systematic) errors. The solid lines are as in Fig. 7. made compatible with DIS and fixed-target experiments, several possibilities have been tried. If one decouples $\Lambda_{\overline{\mathrm{MS}}}$ from $\Lambda\left(\alpha_{s}\right)$ [as in method (b) of Sec. IV], a very good $\chi^{2}$ (about 1 per DF) is obtained with a large value of $\eta_{g}$ $\left(\eta_{g}=7.1\right)$ and a value of $\Lambda\left(\alpha_{s}\right)=0.38 \mathrm{GeV}$ as shown in Fig. 5; this solution is not compatible with either BCDMS or WA70. We also determine the minimum $\chi^{2}$ using the structure functions based on the BCDMS fits of Table I: one finds a value of $\chi^{2}$ of about 3 per DF for $\eta_{g}=4.7$ and $\Lambda_{\overline{\mathrm{MS}}}=0.22 \mathrm{GeV}$, a solution which could be compatible with WA70 if the systematic errors were stretched to the extreme. On the other hand, if the "best" set of structure functions (Appendix B) is kept, but the R806 data are normalized upward by $15 \%$ (which is within the quoted systematic errors) it is found that $\chi_{\mathbf{R} 806}^{2}=40.1$. This is a clear improvement on the value quoted in Table IV, if one realizes, furthermore, that the problem rests now on one point (at $p_{T}=10.5 \mathrm{GeV} / c$ ), with $\chi^{2}=27.5$. If one ignored the difficulty caused by this point, one could conclude that all DP data are consistent among themselves and with BCDMS within their mutual normalization uncertainties.

The results of UA1(Ref. 29) and (Ref. 30) are in relatively good agreement with the theoretical predictions.

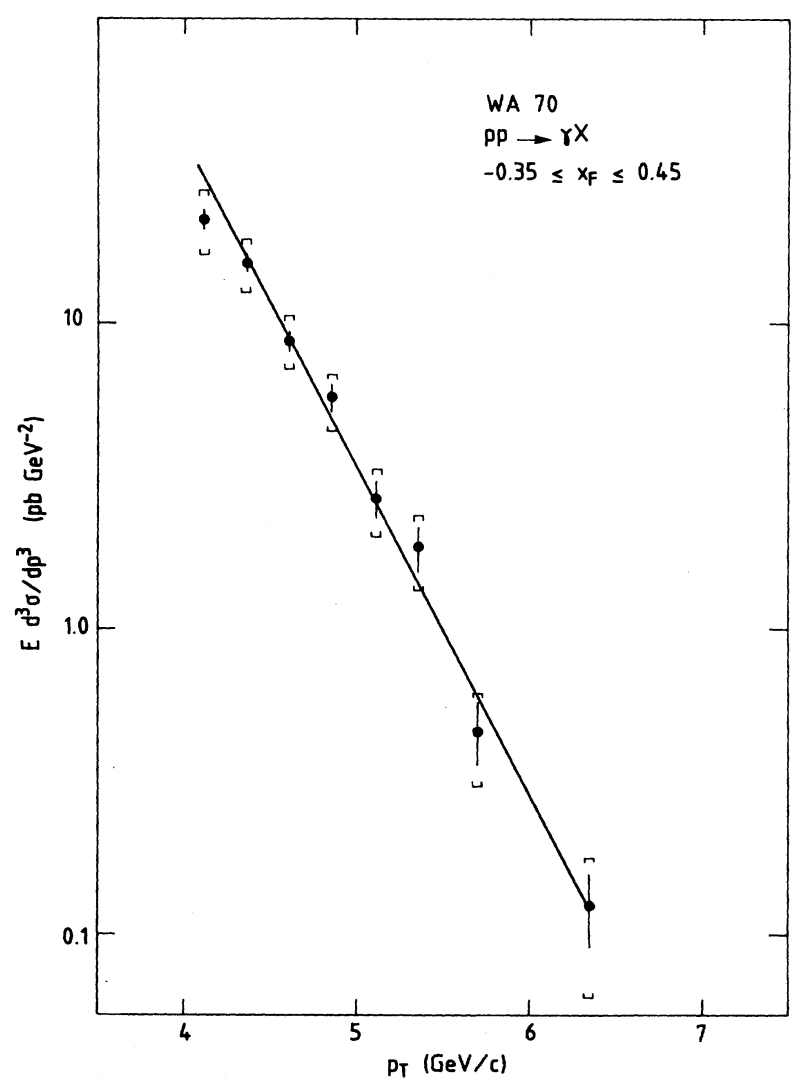

FIG. 9. Cross section of the reaction $p p \rightarrow \gamma X$ (WA70). Statistical error bars are drawn and total errors combining systematic and statistical ones in quadrature are shown. The line is the best fit based on Eq. (10). 


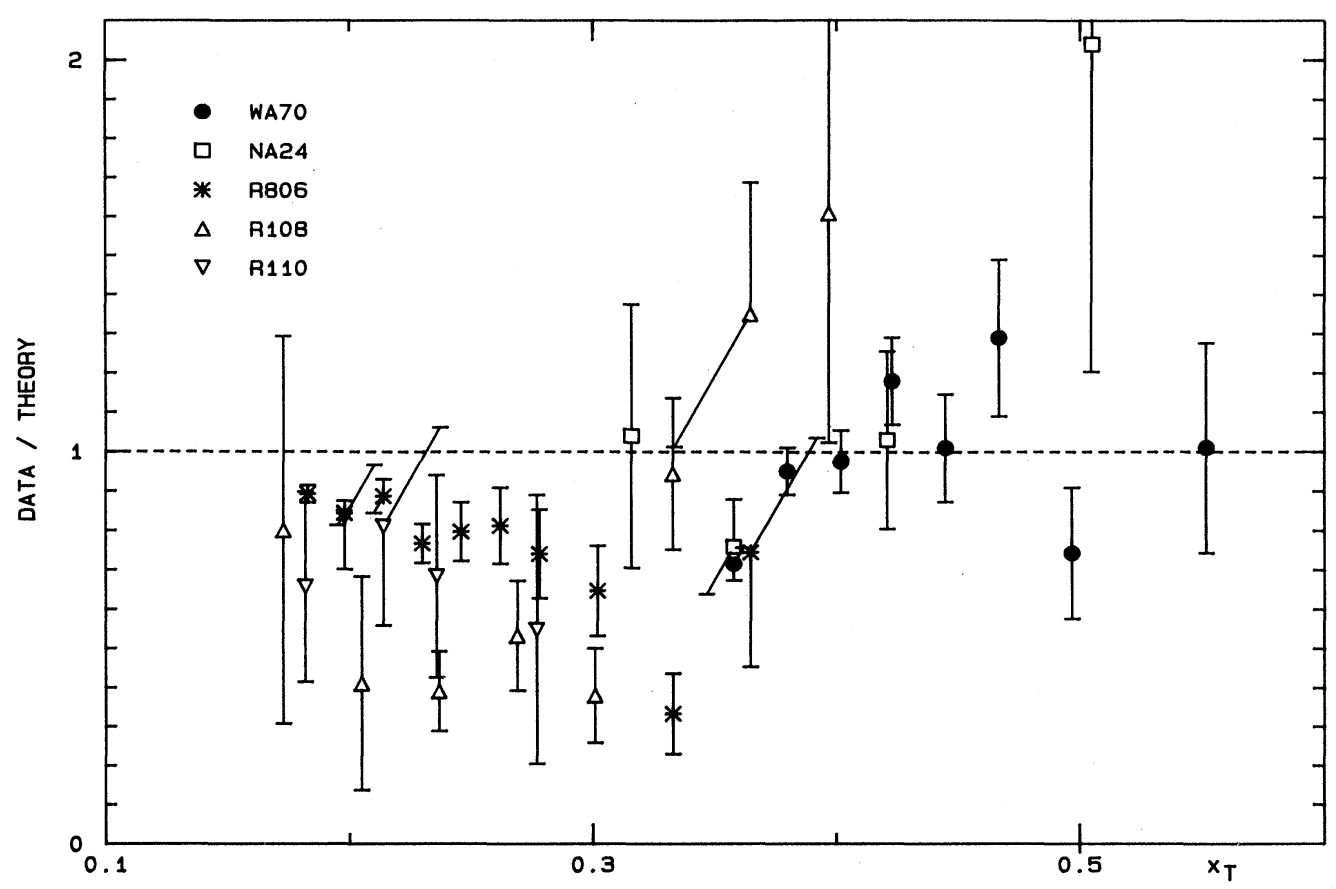

FIG. 10. Ratio data/theory for fixed-target (Refs. 10 and 11) and ISR (Refs. 25-27) $p p \rightarrow \gamma X$ experiments.

In order to reduce the theoretical uncertainties associated with the isolation cuts, we evaluate $\chi^{2}$ for the UA experiments keeping only the data points with $p_{T} \geq 20 \mathrm{GeV}$. We note an appreciable improvement of the values of $\chi^{2}$, as shown in Table $\mathrm{V}$. The UA experiments are, however, quite insensitive to the gluon input shape at $Q_{0}^{2}$ and can- not be used to constrain it in the small- $x$ range. They, however, might allow us to constrain the value of a $\Lambda_{\overline{\mathrm{MS}}}$; a related discussion may be found in Ref. 30 .

From Figs. 10 and 11 we finally observe that the different sets of DP data measured at the different accelerators do not really overlap, when plotted with

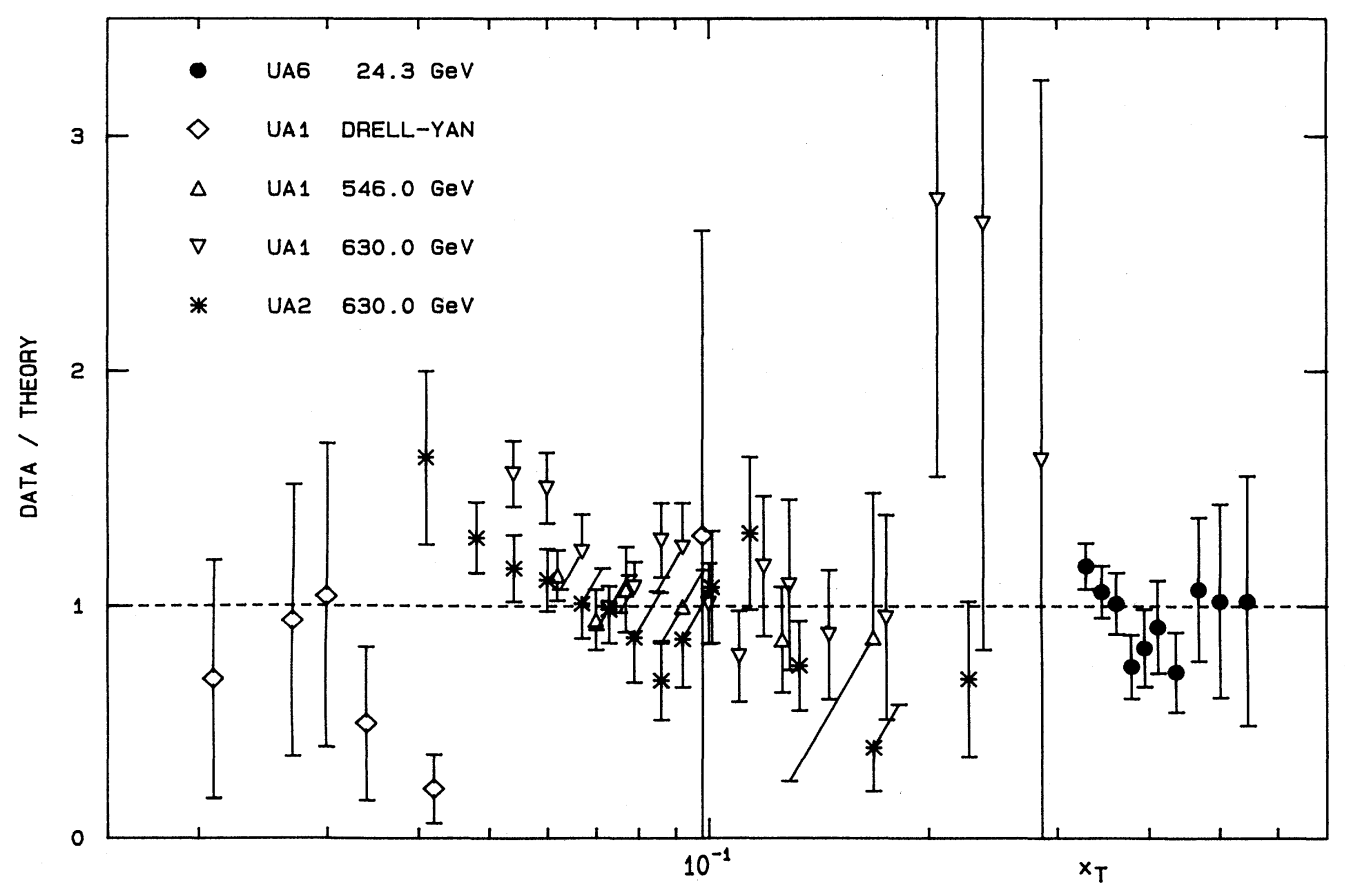

FIG. 11. Ratio data/theory for $p \bar{p} \rightarrow \gamma X$ (Refs. 12 and 28-30). 
TABLE IV. DP data (only statistical errors) compared with ("optimized") QCD DP cross sections. The beyond-leading-order (BLO) $\chi^{2}$ values are obtained with the input equation (10). (Low- $p_{T}$ points for which optimization is not possible are not included.)

\begin{tabular}{|c|c|c|c|c|c|}
\hline Experiment & $\begin{array}{c}\text { Energy } \\
(\mathrm{GeV})\end{array}$ & $\begin{array}{c}\left\langle P_{T}\right\rangle \\
(\mathrm{GeV} / \mathrm{c})\end{array}$ & $\begin{array}{l}\text { Number of } \\
\text { data points }\end{array}$ & DO $1(\mathrm{LO})$ & $\begin{array}{c}\chi^{2} \\
\text { BLO }\end{array}$ \\
\hline \multicolumn{6}{|c|}{$p p \rightarrow \gamma X$} \\
\hline WA70 (Ref. 10) & 23.0 & $4.36-6.34$ & 7 & 58.0 & 7.9 \\
\hline NA24（Ref. 11） & 23.8 & $3.75-6.0$ & 4 & 20.6 & 5.5 \\
\hline R108 (Ref 25) & 62.4 & $5.4-12.4$ & 8 & 134.0 & 80.2 \\
\hline R806 (Ref. 26) & 63.0 & $5.75-11.5$ & 10 & 442.0 & 146.0 \\
\hline R110 (Ref. 27) & 63.0 & $5.72-8.72$ & 5 & 17.5 & 7.4 \\
\hline \multicolumn{6}{|c|}{$p \bar{p} \rightarrow \gamma X$} \\
\hline UA6 (Ref. 12) & 24.3 & $4.0-6.65$ & 10 & 12.4 & 11.0 \\
\hline UA1 (Ref. 28) & 546 & $17.0-46.0$ & 6 & 6.7 & 2.2 \\
\hline UA1 (Ref. 28) & 630 & $17.0-90.0$ & 16 & 64.7 & 39.9 \\
\hline UA2 (Ref. 30) & 630 & $13.0-71.4$ & 14 & 30.7 & 27.5 \\
\hline \multicolumn{6}{|c|}{$p \bar{p} \rightarrow$ (low-mass muon pairs) $X$} \\
\hline UA1 (Ref. 29) & 630 & $6.75-30.75$ & 6 & 18.8 & 31.3 \\
\hline
\end{tabular}

respect to $x_{T}$ (except for the lowest $p_{T}$ data from the fixed-target experiments and the largest- $p_{T}$ data from ISR). Therefore, it would be useful to have DP data at different energies, but with the same values of $x_{T}$, which should be obtained at Fermilab. ${ }^{31,32}$

\section{CONCLUSIONS}

Until now, we have not discussed the theoretical uncertainties associated with the DIS and DP predictions.

It is difficult to estimate the errors we make by not optimizing the perturbative $O\left(\alpha_{s}\right)$ calculation of $F_{2}$. However, the higher-order corrections for DIS structure functions have a weak effect on the determination of the quark distributions and of $\Lambda_{\overline{\mathrm{MS}}}$; beyond-leadinglogarithmic fits are, for instance, very similar to leadinglogarithmic fits.

On the other hand, the determination of the gluon distribution is sensitive to the theoretical inputs. But, as discussed in Sec. III, it is not well constrained by DIS data and must be determined in DP experiments. Therefore, we do not expect a strong effect of the optimization procedure in the present case.

On the contrary, the higher-order corrections in the DP cross sections may be quite large with a strong dependence on the renormalization and factorization masses. We apply the principle of minimal sensitivity ${ }^{13}$ (PMS) criterion to settle this problem; there is, however, no formal proof that this criterion leads to the best predictions. But we can at least say that the optimized cross section becomes locally insensitive to the choice of $M$ and $\mu$. We can also try to use another criterion, the fastest apparent convergence (FAC) criterion. ${ }^{33}$ We found in Ref. 5 that both the PMS and FAC criteria lead to very similar predictions.

Had we used instead the nonoptimized predictions and chosen the scales $M$ and $\mu$ equal to $p_{T}$, the consistent picture described above would have been destroyed: to illustrate this point one can calculate $\chi_{\mathrm{DP}}^{2}$ in the nonoptimized theory for the different DIS fits of Table I. The minimum $\chi_{\mathrm{DP}}^{2}$ are

$$
\chi_{\mathrm{WA} 70}^{2}=13.8 \text { for } \Lambda_{\overline{\mathrm{MS}}}=0.257 \mathrm{GeV} \text { and } \eta_{g}=2.5
$$

and

$\chi_{\mathrm{UA} 6}^{2}=23.2$ for $\Lambda_{\overline{\mathrm{MS}}}=0.272 \mathrm{GeV}$ and $\eta_{g}=2.0$.

Although the $\Lambda_{\overline{\mathrm{MS}}}$ values thus obtained are still consistent within systematic errors with our previous determination, the gluon distribution has become very hard and leads to a very clear degradation of the $\chi_{\mathrm{DP}}^{2}$ values since the $p_{T}$ dependence of the prompt photon spectra cannot be correctly reproduced. Using now the second method of Sec. IV, we introduce $\Lambda\left(\alpha_{s}\right)$ different from $\Lambda_{\overline{\mathrm{MS}}}$ in the structure function and fix the gluon shape at $\eta_{g}=3.9$ which gives the correct $p_{T}$ dependence of the DP cross section. Good agreement with the data is obtained for WA70 if $\Lambda\left(\alpha_{s}\right)=0.6 \mathrm{GeV}\left(\chi_{\text {WA70 }}^{2}=7.6\right)$ and for UA6 if $\Lambda\left(\alpha_{s}\right)=0.5 \mathrm{GeV}\left(\chi^{2}=14\right)$, which is hardly compatible with BCDMS data (even if systematic errors were taken into account). Therefore, it turns out that the very good

TABLE V. Same as Table IV.

\begin{tabular}{lccccr}
\hline \hline Expt. & Energy & $\left\langle P_{T}\right\rangle$ & $\begin{array}{c}\text { Number of } \\
\text { data points }\end{array}$ & $\begin{array}{c}\chi^{2} \\
\text { DO 1 (LO) }\end{array}$ & $\begin{array}{r}\chi^{2} \\
\text { BLO }\end{array}$ \\
\hline UA1 & 546 & $20.0-46.0$ & 4 & 1.3 & 0.6 \\
UA1 & 630 & $20.0-90.0$ & 14 & 23.4 & 12.4 \\
UA2 & 630 & $20.0-71.4$ & 10 & 11.2 & 18.8 \\
\hline \hline
\end{tabular}


TABLE VI. The $\chi_{\text {WA70 }}^{2}$ values as a function of $\eta_{g}$ as defined in Table III.

\begin{tabular}{lrrrrc}
\hline \hline$\eta_{g}$ & 3.5 & 3.8 & 4.0 & 4.5 & 5 \\
Universal & 30.2 & 9.4 & 8.4 & 34.1 & 82.2 \\
Nonuniversal & 30.0 & 9.3 & 8.5 & 35.5 & 85.0 \\
\hline \hline
\end{tabular}

agreement between theory, DIS and DP experiments to a large extent, rests on the optimization procedure.

Bearing in mind the above considerations, we have shown that the DP reactions allow a very precise determination of the gluon distribution. By a common fit to high-statistics DIS data, we have determined a set of beyond-leading-logarithmic quark and gluon distribution functions for $0.05<x<0.75$. We find in particular (cf. Appendix B)

$$
\begin{aligned}
& \eta_{g}=3.90 \pm 0.11\left(\begin{array}{l} 
\pm 0.8 \\
-0.6
\end{array}\right), \\
& \Lambda_{\overline{\mathrm{MS}}}=230.0 \pm 17.0( \pm 50) \mathrm{MeV} .
\end{aligned}
$$

We also observe that the values of $\Lambda_{\overline{\mathrm{MS}}}$, when independently measured in DIS and DP reactions, are in very good agreement.

Note addes in proof. After completion of this work the final data of the BCDMS Collaboration became available (Ref. 35). There are a few minor differences in $F_{2}\left(x, Q^{2}\right)^{\prime}$ at small $x$ and $Q^{2}$ values, but these do not change the quantitative conclusions reported above. For completeness we report, in Appendix C, the results of a fit to these final data.

\section{ACKNOWLEDGMENTS}

We greatly appreciate discussions with L. Camilleri, M.N. Kienzle-Focacci, A. Milsztajn, A. Ouraou, Wu-Ki Tung, and M. Virchaux. One of us (P.A.) thanks A. Martin and the Durham Theory Group for hospitality. The warm hospitality at Laboratoire de Physique de Particules and granting by the Swiss National Fund is acknowledged by M.W. Partial support of this work by "Projets de Coopération et d'échange" (PROCOPE) is kindly acknowledged. Laboratoire de Physique Théorique et Hautes Energies is Laboratoire associé au CNRS.

\section{APPENDIX A}

We discuss here the results of the prompt-photon calculations when one uses the universal or MS definition of the quark distribution. The relation between the universal and the nonuniversal distributions is given by

$$
\begin{aligned}
q^{u}\left(x, Q^{2}\right)=\int_{x}^{l} \frac{d z}{z}[ & \left.\delta(1-z)-\frac{\alpha_{s}}{2 \pi} f_{q}^{(2)}(z) q \mid \frac{x}{z}, Q^{2}\right] \\
& \left.-\frac{\alpha_{s}}{2 \pi} f_{g}^{(2)}(z) g\left(\frac{x}{z}, Q^{2}\right)\right]
\end{aligned}
$$

with $q\left(x, Q^{2}\right)$ defined by Eq. (3) [see Eq. (1) for comparison]. The expression for the prompt-photon cross section has to be changed accordingly when using $q^{u}(x)$ rather than $q(x)$. We perform again the numerical calculations, optimizing with respect to the factorization and the renormalization scales, for the various sets of structure functions defined in Table I. We find very little difference compared to the results based on the nonuniversal quark distributions. Concerning the WA70 kinematical range, for example, the ratio $\sigma\left(q^{u}\right) / \sigma(q)$ is 0.97 at $p_{T}=4.11 \mathrm{GeV}$ and 1.08 at $p_{T}=6.34 \mathrm{GeV}$. The slope of the $p_{T}$ spectrum is very slightly affected but it is a much smaller effect than the precision of the data. Not surprisingly, this uncertainty in the theoretical predictions does not affect the determination of the gluon as can be seen from Table VI which shows $\chi^{2}$ as a function of $\eta_{g}$ for the two choices. In both cases the best $\chi^{2}$ is obtained for $\eta_{g} \simeq 4$.

Similar conclusions can be drawn from the studies of $p p$ reactions at $63 \mathrm{GeV}$ and $p \bar{p}$ reactions at 24 and 630 $\mathrm{GeV}$ : the cross sections never vary by more than $10 \%$ in the kinematical range covered by the data. It is also true at Tevatron energy, tested in the range $10<p_{T}<60$ $\mathrm{GeV} / c$.

These results are an illustration of the consistency of the QCD calculations beyond the leading order.

\section{APPENDIX B}

The full set of parameters [Eq. (4)] corresponding to the best common fit to BCDMS and WA70 data [Eq. (10)] are, at $Q_{0}^{2}=2 \mathrm{GeV}^{2}$,

$$
\begin{aligned}
& \Lambda_{\overline{\mathrm{MS}}}=230.0 \mathrm{MeV} \text { (four flavors), } \eta_{g}=3.90, \\
& A_{g}=1.987, \quad \eta_{1}=0.903, \quad \eta_{2}=3.266, \\
& \gamma_{u d}=-0.680, \quad \eta_{3}=1.053, \quad \eta_{4}=5.179, \\
& A_{s}=1.369, \quad \eta_{S}=10.84 .
\end{aligned}
$$

\section{APPENDIX C}

We present the values of the parameters obtained in a fit based on the final BCDMS ${ }^{35}$ data: $\Lambda_{\overline{\mathrm{MS}}}=231.5 \mathrm{MeV}$ (four flavors), $\quad \eta_{g}=4.00, \quad A_{g}=2.074, \quad \eta_{1}=0.895$, $\eta_{2}=3.173, \quad \gamma_{u d}=-0.745, \quad \eta_{3}=1.050, \quad \eta_{4}=5.207$, $A_{s}=1.120, \eta_{s}=9.374$.

The changes in the values of the parameters, compared to the results in Appendix B, are well within the statistical error bars associated with each parameter.
${ }^{1}$ G. Altarelli, in Proceedings of the International Europhysics Conference on High Energy Physics, Bari, Italy, 1985, edited by L. Nitti and G. Preparata (Laterza, Bari, 1985), p. 729; F. Richard, in Lepton and Photon Interactions, proceedings of the International Symposium on Lepton and Photon Interactions at High Energies, Hamburg, West Germany, 1987, edited by W. Bartel and R. Rück1 [Nucl. Phys. B, Proc. Suppl. 3, 639 (1987)]; L. Camilleri, invited talk at the 19th Symposium 
on Multiparticle Dynamics, Arles, 1988 (unpublished); Report No. CERN-EP/88-125 (unpublished).

${ }^{2}$ Advanced Research Workshop on QCD Hard Hadronic Processes, St. Croix, Virgin Islands, 1987, edited by B. Cox (Plenum, New York, 1989).

${ }^{3}$ For a recent review, see J. F. Owens, Rev. Mod. Phys. 59, 465 (1987).

${ }^{4}$ P. Aurenche, R. Baier, A. Douiri, M. Fontannaz, and D. Schiff, Phys. Lett. 140B, 87 (1984).

${ }^{5}$ P. Aurenche, R. Baier, M. Fontannaz, and D. Schiff, Nucl. Phys. B297, 661 (1988).

${ }^{6}$ D. W. Duke and J. F. Owens, Phys. Rev. D 30, 49 (1984).

${ }^{7} \mathbf{P}$. Aurenche, in Proceedings of the Salt Lake City Meeting, Annual Meeting of the Division of Particles and Fields of the APS, Salt Lake City, Utah, 1987, edited by C. DeTar and J. Ball (World Scientific, Singapore, 1987); R. Baier, in Proceedings of the Seventeenth International Symposium on Multiparticle Dynamics, Seewinkel, Austria, 1986, edited by $\mathbf{M}$. Markytan, W. Majerotto, and J. MacNaughton (World Scientific, Singapore, 1987); and Ref. 2; M. Fontannaz, in Proceedings of the XXIII International Conference on High Energy Physics, Berkeley, California, 1986, edited by S. C. Loken (World Scientific, Singapore, 1987).

${ }^{8}$ BCDMS Collaboration, Phys. Lett. B 195, 91 (1987); 195, 97 (1987); A. Milsztajn, in Proceedings of the European Physical Society High Energy Physics Conference [International Europhysics Conference on High Energy Physics], Uppsala, Sweden, 1987, edited by O. Botner (European Physical Society, Geneva, Switzerland, 1987).

${ }^{9}$ BCDMS Collaboration, presented at the XXIV International Conference on High Energy Physics, Munich, West Germany, 1988 (unpublished); A. Milsztajn (private communication).

${ }^{10}$ WA70 Collaboration, M. Bonesini et al., Z. Phys. C 38, 371 (1988).

${ }^{11}$ NA24 Collaboration, C. De Marzo et al., Phys. Rev. D 36, 8 (1987).

${ }^{12}$ UA6 Collaboration, A. Bernasconi et al., Phys. Lett. B 206, 163 (1988); L. Camilleri (private communication).

${ }^{13}$ P. M. Stevenson, Phys. Rev. D 23, 2916 (1981); H. D. Politzer, Nucl. Phys. B194, 493 (1982); P. M. Stevenson and H D. Politzer, ibid. B277, 758 (1986).

${ }^{14}$ A. Devoto, D. W. Duke, J. F. Owens, and R. G. Roberts, Phys. Rev. D 27, 508 (1983).

${ }^{15}$ EMC Collaboration, J. J. Aubert et al., Nucl. Phys. B259, 189
(1985); B293, 740 (1987).

${ }^{16}$ W. Furmanski and R. Petronzio, Z. Phys. C 11, 293 (1982).

${ }^{17}$ J. F. Owens, Advanced Research Workshop on QCD Hard Hadronic Processes (Ref. 2).

${ }^{18}$ G. Altarelli et al., Nucl. Phys. B153, 52 (1978); B157, 461 (1979).

${ }^{19}$ Wu-Ki Tung, Fermilab Report No. Pub/88-135T (unpublished).

${ }^{20}$ The result is slightly different from the one quoted in M. Werlen, Proceedings of the XXIIIrd Rencontre de Moriond, edited by J. Tran Thanh Van (Editions Frontières, Gif-sur-Yvette, 1988), $\eta_{g}=7.92 \pm 1.57$, which is obtained from an earlier set of preliminary BCDMS data.

${ }^{21}$ A. S. Ito et al., Phys. Rev. D 23, 21 (1981).

${ }^{22}$ D. Antreasyan et al., Phys. Rev. Lett. 48, 302 (1982).

${ }^{23}$ M. Diemoz, F. Ferroni, E. Longo, and G. Martinelli, Z. Phys. C 39, 21 (1988). We thank G. Martinelli for making available to us the results of their latest fits.

${ }^{24}$ CHARM Collaboration, F. Bergsma et al., Phys. Lett. 123B, 269 (1983).

${ }^{25} \mathrm{R} 108$ Collaboration, A. L. S. Angelis et al., Phys. Lett. 94B, 106 (1980); 98B, 115 (1981); L. Camilleri (private communication).

${ }^{26}$ R806 Collaboration, E. Anassontzis et al., Z. Phys. C 13, 277 (1982); C. W. Fabjan and L. Rezvanis (private communication).

${ }^{27}$ R110 Collaboration, C. W. Salgado-Galeazzi, Ph.D dissertation, Michigan State University, 1988.

${ }^{28}$ UA1 Collaboration, C. Albajar et al., Phys. Lett. B 209, 385 (1988).

${ }^{29}$ UA1 Collaboration, C. Albajar et al., Phys. Lett. B 209, 397 (1988). See also P. Aurenche, R. Baier, and M. Fontannaz, ibid. 209, 375 (1988).

${ }^{30}$ UA2 Colloration, R. Ansari et al., Z. Phys. C 41, 395 (1988).

${ }^{31}$ E705 Collaboration, D. Wagoner, Advanced Research on QCD Hard Hadronic Processes (Ref. 2).

${ }^{32}$ E706 Collaboration, G. Fanourakis, Advanced Research on QCD Hard Hadronic Processes (Ref. 2).

${ }^{33}$ G. Grunberg, Phys. Rev. D 29, 2315 (1984).

${ }^{34}$ A. D. Martin, R. G. Roberts, and W. J. Stirling, Phys. Lett. B 206, 327 (1988); Durham Report No. DTP/88/52, 1988 (unpublished).

${ }^{35}$ BCDMS Collaboration, A. C. Benvenuti et al., Reports Nos. CERN-EP/89-06 and CERN-EP/89-07 (unpublished). 